\title{
REGULAR MEASURES AND NORMAL LATTICES
}

\author{
CARMEN VLAD \\ Department of Mathematics \\ Pace University/NYC campus \\ Pace Plaza, New York, NY 10038-1502
}

(Received August 26, 1992 and in revised form December 27, 1992)

\begin{abstract}
In this paper, we investigate $M(\mathcal{L})$ in case $\mathcal{L}$ is a normal lattice of subsets of $X$ and we extend the results to $\ell_{1}, \ell_{2}$-lattices of subsets of $X$, such that $\ell_{1} \subset \ell_{2}$ and $\ell_{1}$ separates $\ell_{2}$. We define the outer measures $\mu^{\prime}$ and $\mu^{\prime \prime}$ which prove very useful in proving some of the above results.
\end{abstract}

KEY WORDS AND PHRASES. Normal lattice, outer measure, separation. 1991 AMS SUBJECT CLASSIFICATION CODES. 28.

\section{INTRODUCTION.}

Let $X$ be an abstract set and $\mathcal{L}$ a lattice of subsets of $X$. If $\mathcal{L}$ is a normal lattice, then in previous papers consequences pertaining to $I(\mathcal{L})$-the set of non-trivial finitely additive zero-one valued measures on $\mathcal{A}(\mathcal{L})$, the algebra generated by $\mathcal{L}$ have been investigated.

In the first part of this paper, we extend these results to $M(\mathcal{L})$, the set of non-trivial, nonnegative finite and finitely additive measures on $\mathcal{A}(\mathcal{L})$. We extend these considerations to $\mathcal{L}_{1}, \mathcal{L}_{2}$ lattices of subsets of $X$ such that $\ell_{1} \subset \ell_{2}$ and $\ell_{1}$ separates $\ell_{2}$.

If $\mu \in M(\mathcal{L})$ then an auxiliary finitely subadditive outer measure $\mu^{\prime}$ is associated with it and proves very useful in proving some of the above results. This along with another outer measure $\mu^{\prime \prime}$ is considered in detail in the second part of the paper. It is shown that although $\mu^{\prime}$ might not be a regular finitely subadditive outer measure, it is still true that an arbitrary set $E \subset X$ is $\mu^{\prime}$ measurable if and only if it splits just $X$ additively. We note that if $\mu \in I(\mathcal{L})$ then $\mu^{\prime}$ is clearly regular, but this need not be the case for $\mu \in M(\ell)$.

We begin with some standard background material (see also [1] and [5]) for the reader's convenience. Some related material can be found in [2], [3], and [4].

\section{BACKGROUND AND NOTATIONS.}

Let $X$ be an abstract set and $\mathcal{L}$ a lattice of subsets of $X$. It is assumed that $\theta, X \in \mathcal{L}$. The lattice $\mathcal{L}$ is called normal if for any $L_{1}, L_{2} \in \mathcal{L}$ with $L_{1} \cap L_{2}=\theta$ there exist $L_{3}, L_{4} \in \mathcal{L}$ with $L_{1} \subset L_{3}^{\prime}, L_{2} \subset L_{4}^{\prime}$ and $L_{3}^{\prime} \cap L_{4}^{\prime}=\theta$ (where prime denotes complement). $\mathcal{L}$ is almost countably compact if $\mu \in I_{R}\left(\mathcal{L}^{\prime}\right)$ implies $\mu \in I_{\sigma}(\mathcal{L})$.

We give now some measure terminology. $M(\mathcal{L})$ denotes the set of finite valued bounded finitely additive measures on $\mathcal{A}(\mathcal{L})$. Without loss of generality may assume throughout that all measures are non-negative. A measure $\mu \in M(\ell)$ is called: 
$\sigma$-smooth on $\mathcal{L}$ if for all sequences $\left\{L_{n}\right\}$ of sets of $\mathcal{L}$ with $L_{n} \downarrow \theta, \mu\left(L_{n}\right) \rightarrow 0$.

$\sigma$-smooth on $\mathcal{A}(\mathcal{L})$ if for all sequences $\left\{A_{n}\right\}$ of sets of $\mathcal{A}(\mathcal{L})$ with $A_{n} \downarrow \theta, \mu\left(A_{n}\right) \rightarrow 0$.

$\mathcal{L}$-regular if for any $A \in \mathcal{A}(\mathcal{L}), \mu(A)=\sup \{\mu(L) / L \subset A, L \in \mathcal{L}\}$.

We denote by $M_{R}(\mathcal{L})$ the set of $\mathcal{L}$-regular measures of $M(\mathcal{L}) ; M_{\sigma}(\mathcal{L})$ the set of $\sigma$-smooth measures on $\mathcal{L}$ of $M(\mathcal{L}) ; M^{\sigma}(\mathcal{L})$ the set of $\sigma$-smooth measures on $\mathcal{A}(\mathcal{L})$ of $M(\mathcal{L}) ; M_{R}^{\sigma}(\mathcal{L})$ the set of $\mathcal{L}$-regular measures of $M^{\sigma}(\mathcal{L})$.

In addition, $I(\mathcal{L}), I_{R}(\mathcal{L}), I_{\sigma}(\mathcal{L}), I^{\sigma}(\mathcal{L})$ and $I_{R}^{\sigma}(\mathcal{L})$ are the subsets of the corresponding $M$ 's which consist of the non-trivial zero-one valued measures.

Finally, for $\ell_{1} \subset \ell_{2}$ two lattices of subsets of $X, \ell_{1}$ separates $\ell_{2}$ if $A, B \in \mathcal{L}_{2}$ and $A \cap B=\theta$ implies there exist $C, D \in \ell_{1}$ such that $A \subset C, B \subset D$ and $C \cap D=\theta$.

\section{NORMAL LATTICES.}

THEOREM 1. Let $\mathcal{\ell}$ be normal and let $\mu \in M(\mathcal{L}), \nu \in M_{R}(\mathcal{L})$ with $\mu \leq \nu$ on $\mathcal{L}$ and $\mu(X)=\nu(X)$. Then $\nu\left(L^{\prime}\right)=\sup \left\{\mu(\widetilde{L}), \widetilde{L} \subset L^{\prime}, L, \widetilde{L} \in \mathcal{L}\right\}$.

PROOF. Let $L \in \mathcal{L}$ and $\varepsilon>0$. Since $\nu \in M_{R}(\mathcal{L})$, there exists $A \subset L^{\prime}, A \in \mathcal{L}$ such that $\nu\left(L^{\prime}\right)-\varepsilon<\nu(A)$. Since $\mathcal{L}$ is normal, there exist $B, C \in \mathcal{L}$ such that $A \subset B^{\prime} \subset C \subset L^{\prime}$. Hence,

$$
\nu(A) \leq \nu\left(B^{\prime}\right) \leq \mu\left(B^{\prime}\right) \leq \mu(C) \leq \nu(C) \leq \nu\left(L^{\prime}\right)
$$

and then $\nu\left(L^{\prime}\right)-\mu(C) \leq \nu\left(L^{\prime}\right)-\nu(A)<\varepsilon$, i.e., $\nu\left(L^{\prime}\right)<\mu(C)+\varepsilon, \varepsilon$ arbitrary small. Therefore, $\nu\left(L^{\prime}\right)=\sup \left\{\mu(\widetilde{L}), \widetilde{L} \subset L^{\prime}, \widetilde{L} \in \mathcal{L}\right\}$.

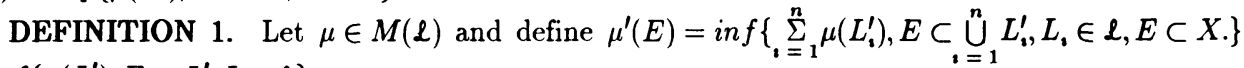
$=\inf \left\{\mu\left(L^{\prime}\right), E \subset L^{\prime}, L \in \ell\right\}$.

THEOREM 2. Suppose $\mathcal{L}$ is normal and let $\mu \in M(\mathcal{L}), \nu \in M_{R}(\mathcal{L})$ with $\mu \leq \nu$ on $\mathcal{L}$ and $\mu(X)=\nu(X)$. Then $\mu \leq \nu=\nu^{\prime}=\mu^{\prime}$ on $\mathcal{L}$.

PROOF. If $A \in \mathcal{L}, \mu(A) \leq \mu\left(L^{\prime}\right)$ for all $A \subset L^{\prime}, L \in \mathcal{L}$. Therefore $\mu(A) \leq \mu^{\prime}(A)=\inf \mu\left(L^{\prime}\right)$, $A \subset L^{\prime}$, i.e., $\mu \leq \mu^{\prime}$ on $\mathcal{L}$. For $\nu \in M_{R}(\mathcal{L})$ we have for any $A \in \mathcal{L}$ :

$$
\nu(A)=\inf \left\{\nu\left(L^{\prime}\right) / A \subset L^{\prime}, L \in \mathcal{L}\right\}=\nu^{\prime}(A)
$$

i.e., $\nu=\nu^{\prime}$ on $\mathcal{L}$. $\mu \leq \nu$ on $\mathcal{L}$ and $\mu(X)=\nu(X)$ implies $\mu \geq \nu$ on $\mathcal{L}^{\prime}$. Hence $\nu^{\prime}(A)=\inf \nu\left(L^{\prime}\right)$ $\leq$ inf $\mu\left(L^{\prime}\right)=\mu^{\prime}(A), A \subset L^{\prime}$, i.e., $\nu^{\prime} \leq \mu^{\prime}$ on $\mathcal{L}$. To show that $\nu^{\prime}=\mu^{\prime}$ on $\mathcal{L}$, suppose that $\nu^{\prime} \neq \mu^{\prime}$ on $\mathcal{L}$. Then there exists $L \in \mathcal{L}$ such that $\nu^{\prime}(L)<\mu^{\prime}(L)$. Since $\nu \in M_{R}(\mathcal{L})$, there exists $A \in \mathcal{L}, L \subset A^{\prime}$ such that $\nu\left(A^{\prime}\right)-\varepsilon<\nu(L)=\nu^{\prime}(L)$.

$\mathcal{L}$ being normal, there exist $C, D \in \mathcal{L}$ such that $L \subset C^{\prime} \subset D \subset A^{\prime}$ and $\mu^{\prime}(L) \leq \mu^{\prime}\left(C^{\prime}\right)$ $\leq \mu\left(C^{\prime}\right) \leq \mu(D)$. But $\mu(D) \leq \nu(D) \leq \nu\left(A^{\prime}\right)<\nu^{\prime}(L)+\varepsilon<\mu^{\prime}(L)+\varepsilon \leq \mu(D)+\varepsilon$, contradiction.

THEOREM 3. Let $\mathcal{L}$ be normal and let $\mu \in M_{\sigma}(\mathcal{L}), \nu \in M_{R}(\mathcal{L})$ with $\mu \leq \nu$ on $\mathcal{L}$ and $\mu(X)=\nu(X)$. Then $\nu \in M_{\sigma}\left(\mathcal{L}^{\prime}\right)$.

PROOF. Let $L_{n}^{\prime} \downarrow \theta, L_{n} \in \mathcal{L}$. Since $\nu \in M_{R}(\mathcal{L})$ and $\mu \in M_{\sigma}(\mathcal{L})$ with $\mu \leq \nu$ on $\mathcal{L}, \nu\left(L^{\prime}\right)=$ sup $\mu(\widetilde{L}), \widetilde{L} \subset L^{\prime} ; L, \widetilde{L} \in \mathcal{L}$. Given $\varepsilon>0$, there exist $\tilde{L}_{n} \subset L_{n}^{\prime}, \tilde{L}_{n} \in \mathcal{L}$ such that $\nu\left(L_{n}^{\prime}\right)<\mu\left(\tilde{L}_{n}\right)+\frac{\varepsilon}{2}$. May assume $\tilde{L}_{n} \downarrow$ and since $\tilde{L}_{n} \subset L_{n}^{\prime} \downarrow \theta$ it follows $\tilde{L}_{n} \downarrow \theta$. Since $\mu \in M_{\sigma}(\mathcal{L}), \mu\left(\tilde{L}_{n}\right)<\frac{\varepsilon}{2}$, all $n \geq N(\varepsilon)$. Hence $\nu\left(L_{n}^{\prime}\right)<\varepsilon$ for all $n \geq N(\varepsilon)$, i.e., $\nu \in M_{\sigma}\left(\ell^{\prime}\right)$.

THEOREM 4. Suppose $\mathcal{L}$ is normal and let $\mu \in M(\mathcal{L}), \nu_{1}, \nu_{2} \in M_{R}(\mathcal{L})$ with $\mu \leq \nu_{1}$ on $\mathcal{L}$, $\mu \leq \nu_{2}$ on $\mathcal{L}$ and $\mu(X)=\nu_{1}(X)=\nu_{2}(X)$. Then $\nu_{1}=\nu_{2}$.

PROOF. By Theorem 2,

$$
\mu \leq \nu_{1}=\nu_{1}^{\prime}=\mu^{\prime}, \quad \mu \leq \nu_{2}=\nu_{2}^{\prime}=\mu^{\prime} \text { on } \mathcal{L} .
$$


Hence $\nu_{1}=\nu_{2}=\mu^{\prime}$

THEOREM 5. Let $\mathcal{L}$ be almost countably compact and let $\mu \in M_{R}\left(\mathcal{L}^{\prime}\right)$. Then $\mu \in M_{\sigma}(\mathcal{L})$.

PROOF. Suppose $\mu \notin M_{\sigma}(\mathcal{L})$. Then there exists $A_{n} \downarrow \theta, A_{n} \in \mathcal{L}$ with $\mu\left(A_{n}\right) \neq 0$. Since $\mu \in M_{R}\left(\mathcal{L}^{\prime}\right)$, there exists $B_{n} \in \mathcal{L}, A_{n} \supset B_{n}^{\prime}$ and $0 \leftarrow \mu\left(A_{n}\right) \sim \mu\left(B_{n}^{\prime}\right)$. May assume $B_{n}^{\prime} \neq \theta$ all $n$ and $B_{n}^{\prime} \downarrow \emptyset$, hence $\left\{B_{n}^{\prime}\right\}$ has the finite intersection property. Therefore there exists $\lambda \in I_{R}\left(\mathcal{L}^{\prime}\right)$ such that $\lambda\left(B_{n}^{\prime}\right)=1$ all $n$. Since $A_{n} \supset B_{n}^{\prime}$ it follows $\lambda\left(A_{n}\right)=1$ all $n$. But $A_{n} \downarrow \theta$; then $\lambda \notin I_{\sigma}(\ell)$, contradiction since $\mathcal{L}$ is almost countably compact.

THEOREM 6. Let $\ell_{1} \subset \ell_{2}$ and suppose that $\ell_{1}$ separates $\ell_{2}$. Let $\mu \in M_{R}\left(\ell_{1}\right)$ and consider the extension $\nu \in M_{R}\left(\ell_{2}\right)$. Then:

(a) $\nu$ is $\ell_{1}$-regular on $\ell_{2}^{\prime}$.

(b) If $\mu \in M_{R}^{\sigma}\left(\ell_{1}\right)$ then $\nu \in M_{\sigma}\left(\ell_{2}^{\prime}\right)$.

(c) $\nu$ is unique.

PROOF.

(a) Define $\mu_{\mathrm{i}}\left(L_{2}\right)=\inf \mu\left(L_{1}^{\prime}\right), L_{2} \subset L_{1}^{\prime}, L_{2} \in \ell_{2}, L_{1} \in \ell_{1}$. For any $L_{1}^{\prime} \supset L_{2}$ we have $\nu\left(L_{2}\right)$ $\leq$ inf $\mu\left(L_{1}^{\prime}\right)=\mu_{1}\left(L_{2}\right)$, hence $\nu \leq \mu_{1}$ on $\ell_{2}$. Suppose that $\nu\left(L_{2}\right)<\mu_{1}\left(L_{2}\right)$ for some $L_{2} \in \ell_{2}$. Since $\nu \in M_{R}\left(\ell_{2}\right)$, there exists $\left.\tilde{L}_{2} \in \ell_{2}, L_{2} \subset \tilde{L}_{2}^{\prime}\right)$ such that $\nu\left(\tilde{L}_{2}^{\prime}\right)<\nu\left(L_{2}\right)+\varepsilon$. By separation, there exist $L_{1}, \tilde{L}_{1} \in \mathcal{L}_{1}$ such that $L_{2} \subset L_{1}, \tilde{L}_{2} \subset \tilde{L}_{1} \quad$ and $L_{1} \cap \tilde{L}_{1}=\emptyset$. Then $L_{2} \subset L_{1} \subset \tilde{L}_{1}^{\prime} \subset \tilde{L}_{2}^{\prime}$ and $\nu\left(L_{2}\right)<\nu\left(L_{1}\right)=\mu\left(L_{1}\right)<\nu\left(\tilde{L}_{2}^{\prime}\right)<\mu_{\mathrm{s}}\left(L_{2}\right)+\varepsilon, \varepsilon$ arbitrary small. It follows $\mu\left(L_{1}\right)<\mu_{\mathrm{s}}\left(L_{2}\right)$. But $L_{2} \subset L_{1}$ implies $\mu_{2}\left(L_{2}\right) \leq \mu\left(L_{1}\right)$, contradiction. Hence we must have $\nu=\mu_{\mathrm{t}}$ on $\ell_{2}$ or $\nu=\mu_{*}$ on $\ell_{2}^{\prime}$.

(b) Let $L_{n}^{\prime} \in \mathcal{L}_{2}^{\prime}, L_{n}^{\prime} \downarrow \theta . \quad \nu\left(L_{n}^{\prime}\right)=\mu_{*}\left(L_{n}^{\prime}\right)=\sup \left\{\mu\left(\tilde{L}_{n}\right), \tilde{L}_{n} \subset L_{n}^{\prime}, \tilde{L}_{n} \in \mathcal{L}_{1}\right\}$. Since $L_{n}^{\prime} \downarrow \theta$ and $\tilde{L}_{n} \subset L_{n}^{\prime}$, may assume $\tilde{L}_{n} \downarrow \theta$. Given $\varepsilon>0$, there exists $\tilde{L}_{n} \subset L_{n}^{\prime}$ such that $\nu\left(L_{n}^{\prime}\right)-\varepsilon<\mu\left(\tilde{L}_{n}\right)$. $\mu \in M_{R}^{\sigma}\left(\ell_{1}\right)$ implies $\mu\left(\tilde{L}_{n}\right) \rightarrow 0$, hence $\nu\left(L_{n}^{\prime}\right) \rightarrow 0$, i.e., $\nu \in M_{\sigma}\left(\ell_{2}^{\prime}\right)$.

(c) Suppose for $\mu \in M_{R}^{\sigma}\left(\ell_{1}\right)$ there are two extensions $\nu_{1}, \nu_{2} \in M_{R}\left(\ell_{2}\right)$. By (a) $\nu_{1}, \nu_{2}$, are $\ell_{1}$-regular on $\ell_{2}^{\prime}$, i.e.,

$$
\begin{aligned}
& \nu_{1}\left(L_{2}^{\prime}\right)=\sup \mu\left(L_{1}\right), L_{1} \subset L_{2}^{\prime}, L_{1} \in \ell_{1}, L_{2} \in \ell_{2} . \\
& \nu_{2}\left(L_{2}^{\prime}\right)=\sup \mu\left(L_{1}\right), L_{1} \subset L_{2}^{\prime}, L_{1} \in \ell_{1}, L_{2} \in \ell_{2} .
\end{aligned}
$$

Hence $\nu_{1}\left(L_{2}^{\prime}\right)=\nu_{2}\left(L_{2}^{\prime}\right)$ and then $\nu_{1}=\nu_{2}$ on $\ell_{2}^{\prime}$ which implies $\nu_{1}=\nu_{2}$ on $\ell_{2}$.

THEOREM 7. Let $\ell_{1} \subset \ell_{2}$ and suppose $\ell_{1}$ separates $\ell_{2}$. Let $\mu \in M\left(\ell_{1}\right), \nu \in M_{R}\left(\ell_{1}\right)$ with $\mu \leq \nu$ on $\ell_{1}$ and $\mu(X)=\nu(X)$. Consider the extensions $\tau \in M\left(\ell_{2}\right), \tau / \mathcal{\Lambda}_{\left(\ell_{1}\right)}=\mu$ and $\lambda \in M_{R}\left(\ell_{2}\right)$, $\lambda /{ }_{a\left(\ell_{1}\right)}=\nu$. Then $\tau \leq \lambda$ on $\ell_{2}$.

PROOF. Let $L_{2} \in \mathcal{L}_{2}$, arbitrary. Since $\lambda$ is $\ell_{2}$-regular, given $\varepsilon>0$, there exists $\tilde{L}_{2} \in \ell_{2}$, $L_{2} \subset \tilde{L}_{2}^{\prime}$ such that $\lambda\left(\tilde{L}_{2}^{\prime}\right)<\lambda\left(L_{2}\right)+\varepsilon$. By separation, there exists $L_{1}, \tilde{L}_{1} \in \ell_{1}$ such that $L_{2} \subset L_{1}$, $\tilde{L}_{2} \subset \tilde{L}_{1}$ and $L_{1} \cap \tilde{L}_{1}=\theta$. Therefore, we have $L_{2} \subset L_{1} \subset \tilde{L}_{1}^{\prime} \subset \tilde{L}_{2}^{\prime}$ and $\tau\left(L_{2}\right) \leq \tau / \mu_{\left(\ell_{1}\right)}\left(L_{1}\right)=\mu\left(L_{1}\right)$ $\leq \nu\left(L_{1}\right) \leq \nu\left(\tilde{L}_{1}^{\prime}\right)=\lambda /_{\mathcal{\Lambda}\left(\ell_{1}\right)}\left(\tilde{L}_{1}^{\prime}\right) \leq \lambda\left(\tilde{L}_{2}^{\prime}\right)<\lambda\left(L_{2}\right)+\varepsilon$ and $\lambda=\nu$ on $\ell_{1}, \ell_{1}^{\prime} ; \varepsilon$ arbitrary small implies $\tau\left(L_{2}\right) \leq \lambda\left(L_{2}\right) . \quad L_{2}$ arbitrary in $\ell_{2}$ shows that $\tau \leq \lambda$ on $\ell_{2}$.

THEOREM 8. Let $\mathcal{L}$ be normal and almost countably compact. Then $M_{R}(\mathcal{L}) \subset M_{\sigma}\left(\mathcal{L}^{\prime}\right)$.

PROOF. Let $\mu \in M_{R}(\mathcal{L})$. Then $\mu \leq \rho \in M_{R}\left(\mathcal{L}^{\prime}\right)$ on $\mathcal{L}^{\prime}$ and $\mu(X)=\rho(X)$. Hence $\rho \leq \mu$ on $\mathcal{L}$ and $\rho \in M_{\sigma}(\mathcal{L})$ since $\mathcal{L}$ is almost countably compact. But $\rho \in M_{\sigma}(\mathcal{L})$ and $\mu \in M_{R}(\mathcal{L})$ and $\rho \leq \mu$ on $\mathcal{L}$, therefore, $\mathcal{L}$ being normal it follows $\mu \in M_{\sigma}\left(\mathcal{L}^{\prime}\right)$.

\section{SOME FINITELY SUBADDITIVE OUTER MEASURES.}

DEFINITION 2. $\mu$ defined on $\Phi(X)$ is a finitely subadditive outer measure if:

(a) $\mu$ is nondecreasing;

(b) $\mu\left(\bigcup_{\imath=1}^{n} E_{\imath}\right) \leq \sum_{\imath=1}^{n} \mu\left(E_{\imath}\right)$, for any $E_{1}, E_{2}, \cdots E_{n} \in X$; 
(c) $\mu(\theta)=0$

Clearly, $\mu^{\prime}$, as defined in Section 3 , is a finitely subadditive outer measure.

Let $\varphi_{\mu^{\prime}}$ be the set of all $\mu^{\prime}$-measurable sets, where $E$ is measurable with respect to $\mu^{\prime}$ if for any $A \subset X, \mu^{\prime}(A)=\mu^{\prime}(A \cap E)+\mu^{\prime}\left(A \cap E^{\prime}\right)$

THEOREM 9. $E \subset \mathcal{\varphi}_{\mu^{\prime}}$ if and only if $\mu^{\prime}\left(A^{\prime}\right) \geq \mu^{\prime}\left(A^{\prime} \cap E\right)+\mu^{\prime}\left(A^{\prime} \cap E^{\prime}\right)$ for all $A \in \mathcal{L}$.

\section{PROOF.}

(a) If $E \subset \varphi_{\mu^{\prime}}$, then the relation clearly holds.

(b) Let $B$ be arbitrary in $X, B \subset A^{\prime}, A \in \mathcal{L}$. We have:

$$
\mu\left(A^{\prime}\right)=\mu^{\prime}\left(A^{\prime}\right) \geq \mu^{\prime}\left(A^{\prime} \cap E\right)+\mu^{\prime}\left(A^{\prime} \cap E^{\prime}\right) \geq \mu^{\prime}(B \cap E)+\mu^{\prime}\left(B \cap E^{\prime}\right)
$$

by assumption and $\mu^{\prime}$ being monotone. Since this is true for any $B \subset A^{\prime}, A \in \mathcal{L}$, it follows $\mu^{\prime}(B) \geq \mu^{\prime}(B \cap E)+\mu^{\prime}\left(B \cap E^{\prime}\right)$. By the definition of $\mu^{\prime}$ as an outer measure, we have for $B=(B \cap E) U\left(B \cap E^{\prime}\right): \mu^{\prime}(B) \leq \mu^{\prime}(B \cap E)+\mu^{\prime}\left(B \cap E^{\prime}\right)$. Therefore, $\mu^{\prime}(B)=\mu^{\prime}(B \cap E)+\mu^{\prime}\left(B \cap E^{\prime}\right)$, $B$ arbitrary in $X$, i.e., $E \in \mathcal{I}_{\mu^{\prime}}$.

DEFINITION 3. Let $\mu \in M(\ell)$ and define the inner measure $\mu_{\imath}(E)=\sup \mu(L)$, $L \subset E, L \in \mathcal{L}, E \subset X$.

THEOREM 10. The following statements are true:

(a) $\mu(X)=\mu_{\imath}(L)+\mu^{\prime}\left(L^{\prime}\right), L \in \mathcal{L}$.

(b) $\mu(X)=\mu_{1}\left(L^{\prime}\right)+\mu^{\prime}(L)$

PROOF. Clear.

DEFINITION 4. Let $\mu \in M_{\sigma}(\mathcal{L}), E \subset X$ and define $\mu^{\prime \prime}(E)=\inf \sum_{i=1}^{\infty} \mu\left(L_{1}^{\prime}\right), E \subset \bigcup_{i=1}^{\infty} L_{n}^{\prime}, L_{\mathfrak{t}} \in \mathcal{L}$. Let $\varphi_{\mu^{\prime \prime}}$ be the set of $\mu^{\prime \prime}$-measurable sets, where $E$ is measurable with respect to $\mu^{n} \bar{n}^{1}$ if for any $A \subset X \mu^{\prime \prime}(A)=\mu^{\prime \prime}(A \cap E)+\mu^{\prime \prime}\left(A \cap E^{\prime}\right)$.

THEOREM 11. $\mu^{\prime \prime}$ is an outer measure.

PROOF. Clear.

THEOREM 12. $E \in \mathcal{Y}_{\mu^{\prime \prime}}$ if and only if $\mu^{\prime \prime}\left(A^{\prime}\right) \geq \mu^{\prime \prime}\left(A^{\prime} \cap E\right)+\mu^{\prime \prime}\left(A^{\prime} \cap E^{\prime}\right)$ for all $A \in \mathcal{L}$. PROOF.

(a) If $E \in \mathcal{Y}_{\mu^{\prime \prime}}$, then clearly $\mu^{\prime \prime}\left(A^{\prime}\right) \geq \mu^{\prime \prime}\left(A^{\prime} \cap E\right)+\mu^{\prime \prime}\left(A^{\prime} \cap E^{\prime}\right)$ for $A \in \mathcal{L}$.

(b) Let $B$ be arbitrary set in $X$ and let $B \subset \bigcup_{i=1}^{\infty} L_{1}^{\prime}, L_{1} \in \mathcal{L}$ all $i$. Then, since $\mu^{\prime \prime} \leq \mu$ on $\mathcal{L}^{\prime}$, we have

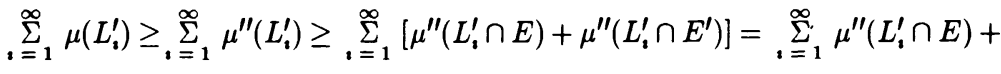

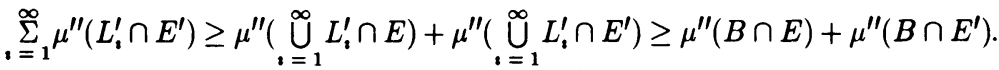

This holds for all $B, B \subset \bigcup_{i=1}^{\infty} L_{\mathfrak{v}}^{\prime}$, therefore $\mu^{\prime \prime}(B)=\inf f_{i=1}^{\infty} \mu\left(L_{\mathfrak{\imath}}^{\prime}\right) \geq \mu^{\prime \prime}(B \cap E)+\mu^{\prime \prime}\left(B \cap E^{\prime}\right)$ and since $B$ was arbitrary in $X$ it follows $E \in \varphi_{\mu^{\prime \prime}}$.

THEOREM 13. Let $\mu \in M(\mathcal{L}), E \subset X . \quad E \in \mathcal{\varphi}_{\mu^{\prime}}$ iff $\mu_{\mathfrak{\imath}}(E)=\mu^{\prime}(E)$.

PROOF. Suppose $E \in \mathcal{\varphi}_{\mu^{\prime}}$. Then $\mu(X)=\mu^{\prime}\left(E^{\prime}\right)+\mu^{\prime}(E)$. By Theorem 10, $\mu(X)=\mu_{\mathrm{s}}\left(E^{\prime}\right)$ $+\mu^{\prime}(E)$, so we have $\mu^{\prime}\left(E^{\prime}\right)=\mu_{\mathrm{v}}\left(E^{\prime}\right)$. Hence $\mu(X)-\mu^{\prime}\left(E^{\prime}\right)=\mu(X)-\mu_{\mathrm{v}}\left(E^{\prime}\right)$ and then $\mu_{\mathfrak{s}}(E)=\mu^{\prime}(E)$. Conversely, suppose $\mu_{\mathfrak{\imath}}(E)=\mu^{\prime}(E)$. Then, given $\varepsilon>0$, there exists $\widehat{L} \in \mathcal{L}, \widehat{L} \subset E$ and $\mu(\widehat{L})+\frac{\varepsilon}{2}>\mu(E)$. Also, be definition of $\mu^{\prime}$, there exists $\widetilde{L} \in \mathcal{L}$ such that $\tilde{L}^{\prime} \supset E \supset \widehat{L}$ and $\mu\left(\tilde{L}^{\prime}\right)<\mu^{\prime}(E)+\frac{\varepsilon}{2}$. Now, let $A^{\prime} \in \mathcal{L}^{\prime}$. Then $\mu^{\prime}\left(A^{\prime} \cap E\right) \leq \mu^{\prime}\left(A^{\prime} \cap \tilde{L}^{\prime}\right)=\mu\left(A^{\prime}\right)+\mu\left(\tilde{L}^{\prime}\right)-\mu\left(A^{\prime} U \tilde{L}^{\prime}\right)$ $\leq \mu\left(A^{\prime}\right)+\mu^{\prime}(E)+\frac{\varepsilon}{2}-\mu\left(A^{\prime} U \widehat{L}\right) \leq \mu\left(A^{\prime}\right)+\mu(\widehat{L})+\frac{\varepsilon}{2}+\frac{\varepsilon}{2}-\mu\left(A^{\prime} U \widehat{L}\right)=\mu\left(A^{\prime} \cap \widehat{L}\right)+\varepsilon . \quad$ Now $\quad E^{\prime} \subset \widehat{L}^{\prime}$, hence $A^{\prime} \cap E^{\prime} \subset A^{\prime} \cap \widehat{L}^{\prime}$. Thus $\mu^{\prime}\left(A^{\prime} \cap E\right)+\mu^{\prime}\left(A^{\prime} \cap E^{\prime}\right) \leq \mu\left(A^{\prime} \cap \widehat{L}\right)+\varepsilon+\mu^{\prime}\left(A^{\prime} \cap \widehat{L}^{\prime}\right)=\mu\left(A^{\prime} \cap \widehat{L}\right)$ 
$+\varepsilon+\mu\left(A^{\prime} \cap \widehat{L}^{\prime}\right)=\mu\left(A^{\prime}\right)+\varepsilon, \varepsilon$ arbitrary.

Therefore, $\mu^{\prime}\left(A^{\prime} \cap E\right)+\mu^{\prime}\left(A^{\prime} \cap E^{\prime}\right) \leq \mu\left(A^{\prime}\right)=\mu^{\prime}\left(A^{\prime}\right), A^{\prime} \in \mathcal{L}^{\prime}$, i.e., $E \in \mathcal{Y}_{\mu^{\prime}}$.

THEOREM 14. Let $\mu \in M_{\sigma}(\mathcal{L})$ and define $\mu^{\prime \prime}(E)$ as above. Then

(a) $\mu^{\prime \prime}(X)=\mu(X)$

(b) $\mu \leq \mu^{\prime \prime}$ on $\mathcal{L}$.

\section{PROOF.}

(a) If $\mu^{\prime \prime}(X) \neq \mu(X)$, there exists $L_{\imath} \in \mathcal{L}, \imath=1,2, \cdots$ such that $X=\bigcup_{i=1}^{\infty} L_{1}^{\prime}$ and

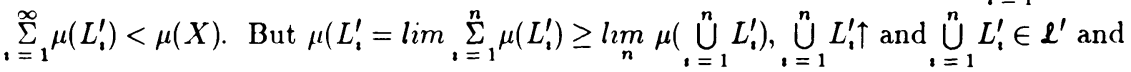

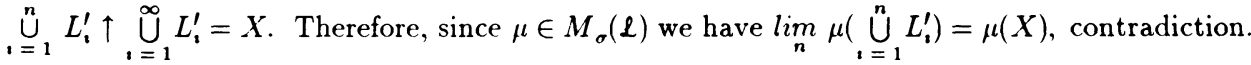

(b) Suppose there exists $L \in \mathcal{L}$ such that $\mu(L)>\mu^{\prime \prime}(L)$. Then $\mu^{\prime \prime}(X) \leq \mu^{\prime \prime}(L)+\mu^{\prime \prime}\left(L^{\prime}\right)$ $\leq \mu^{\prime \prime}(L)+\mu\left(L^{\prime}\right)<\mu(L)+\mu\left(L^{\prime}\right)=\mu(X)$ which contradicts part (a) of the theorem.

\section{REFERENCES}

1. ALEXANDROFF, A.D., Additive set functions in abstract spaces, Mat. Sb. (N.S.) $8(50)$ (1940), 307-348.

2. BACHMAN, G. \& SULTAN, A., Regular lattice measures: mappings and spaces, Pac. J. Math. 67 (2) (1976), 291-321.

3. EID, G., On normal lattices and Wallman spaces, Internat. J. Math. and Math. Sci. 13 (1) (1990), 31-38.

4. SZETO, M., On separation of lattices, Internat. J. Math. and Math. Sci. 14 (2) (1991), 325338.

5. VLAD, C., On normal lattices and semiseparation of lattices, The Journal of the Indian Mathematical Society, Panjab University of India, Vol. 56 (1991), 259-273. 


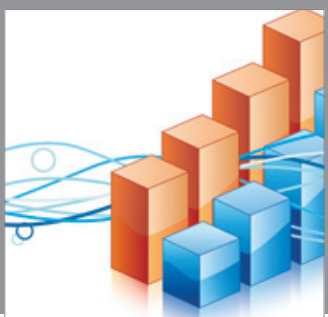

Advances in

Operations Research

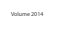

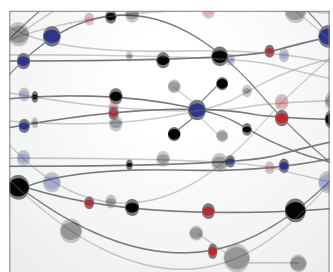

\section{The Scientific} World Journal
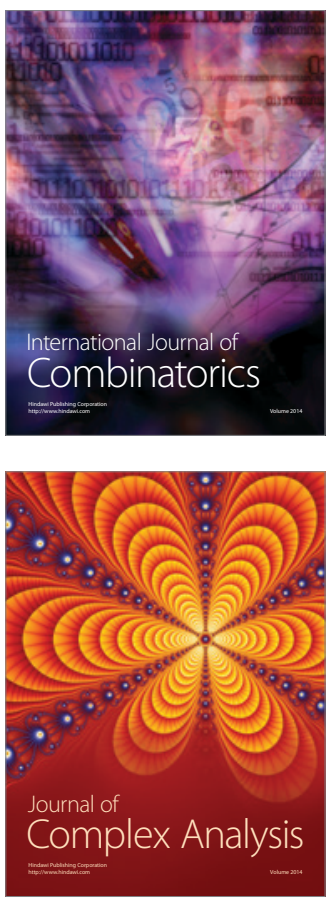

International Journal of

Mathematics and

Mathematical

Sciences
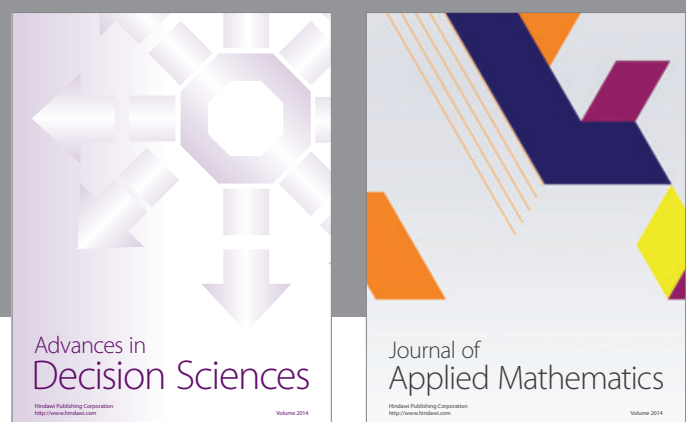

Journal of

Applied Mathematics
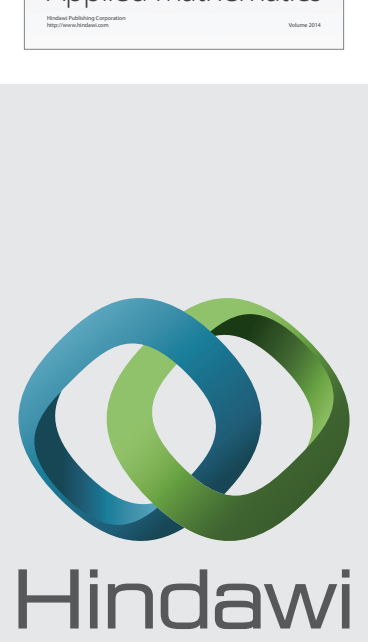

Submit your manuscripts at http://www.hindawi.com
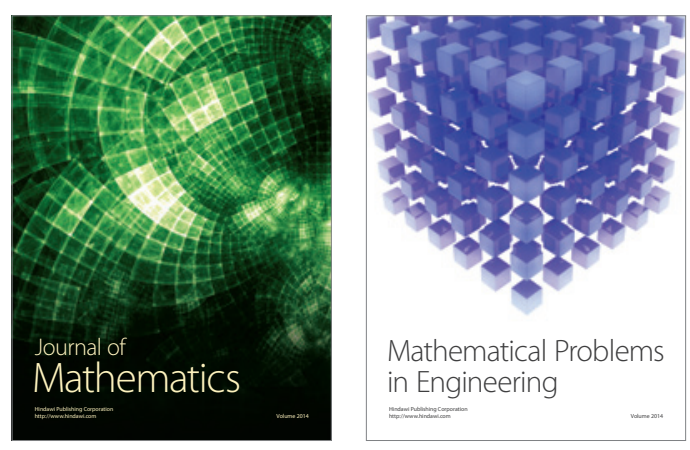

Mathematical Problems in Engineering
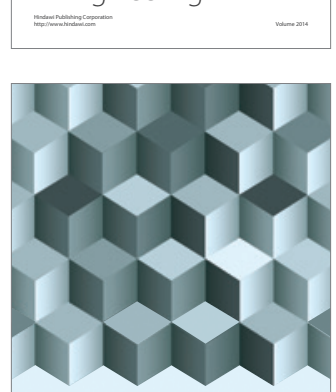

Journal of

Function Spaces
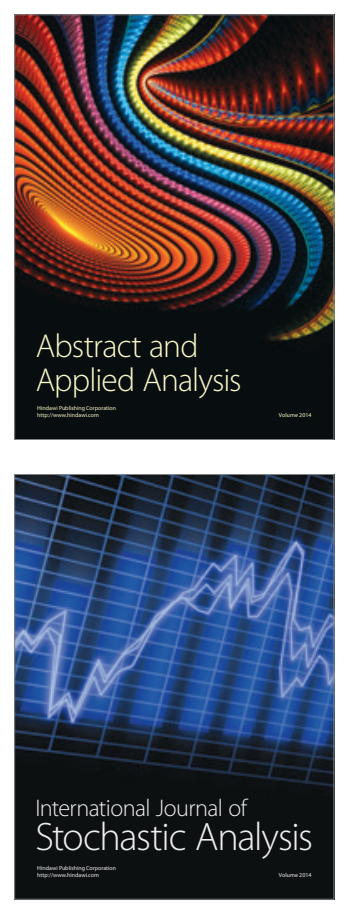

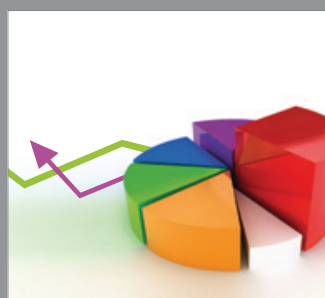

ournal of

Probability and Statistics

Promensencen
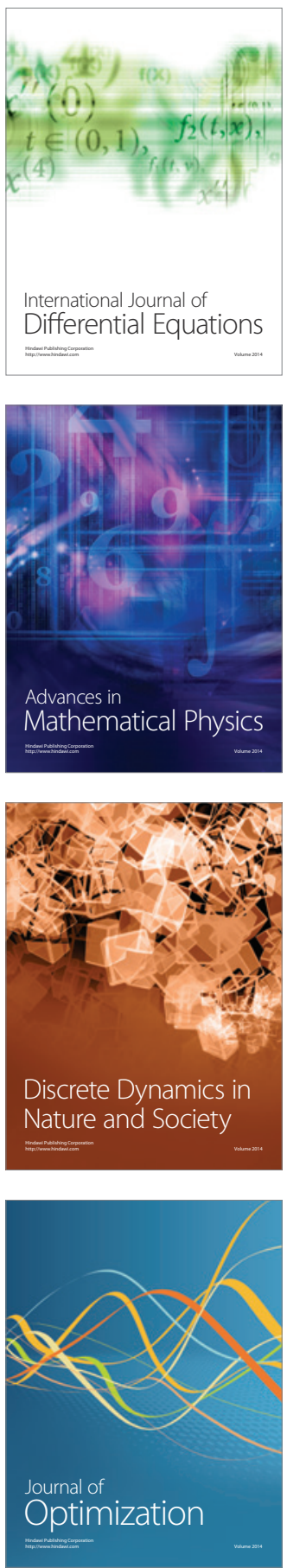